\title{
One-dimensional Kardar-Parisi-Zhang and Kuramoto-Sivashinsky universality class: Limit distributions
}

\author{
Dipankar Roy $\oplus^{*}$ \\ Department of Mathematics, Indian Institute of Science, Bangalore 560012, India \\ Rahul Pandit ${ }^{\dagger}$ \\ Centre for Condensed Matter Theory, Department of Physics, Indian Institute of Science, Bangalore 560012, India
}

(Received 29 August 2019; accepted 10 March 2020; published 27 March 2020)

\begin{abstract}
Tracy-Widom and Baik-Rains distributions appear as universal limit distributions for height fluctuations in the one-dimensional Kardar-Parisi-Zhang (KPZ) stochastic partial differential equation (PDE). We obtain the same universal distributions in the spatiotemporally chaotic, nonequilibrium, but statistically steady state of the one-dimensional Kuramoto-Sivashinsky (KS) deterministic PDE, by carrying out extensive pseudospectral direct numerical simulations to obtain the spatiotemporal evolution of the KS height profile $h(x, t)$ for different initial conditions. We establish, therefore, that the statistical properties of the one-dimensional (1D) KS PDE in this state are in the $1 \mathrm{D} \mathrm{KPZ} \mathrm{universality} \mathrm{class.}$
\end{abstract}

DOI: 10.1103/PhysRevE.101.030103

Fundamental investigations of the statistical properties of hydrodynamical turbulence often use randomly forced versions of the deterministic Navier-Stokes (NS) equations (3 D NSE, in three dimensions); the latter use a nonrandom forcing term to produce a turbulent, but nonequilibrium, statistically steady state (NESS). A randomly forced 3D, incompressible NS equation (3 D RFNSE), first proposed by Edwards [1] in 1964, has been studied extensively, via renormalization-group (RG) and other theoretical [2-9] and numerical [10,11] methods; these studies have shown that many statistical properties of turbulence in the 3D RFNSE are akin to their 3D NSE counterparts. In particular, the wave-number $k$ dependence of the energy spectrum [12-14] $E(k)$, and even the multiscaling corrections [14-18] to the Kolmogorov phenomenology [12-14] of 1941 are similar in both these models.

Can we find such a similarity between the statistical properties of NESSs in deterministic and related stochastic partial differential equations (PDEs) that are simpler than their 3D hydrodynamical counterparts? It has been suggested, since the 1980s, that the Kuramoto-Sivashinsky (KS) PDE, a deterministic interface-growth model for a height field $h(\mathbf{x}, t)$, which is used in studies of chemical waves, flame fronts, and the surfaces of thin films flowing under gravity [19-25], is a simplified model for turbulence [23]. It has been conjectured [26], and subsequently shown by compelling numerical studies [27-32], in both one dimension (1D) and two dimensions (2D), that the long-distance and long-time behaviors of correlation functions, in the spatiotemporally chaotic NESS of the KS PDE, exhibit the same power-law scaling as their counterparts in the the Kardar-Parisi-Zhang

\footnotetext{
*dipankarroy@iisc.ac.in

${ }^{\dagger}$ Also at Jawaharlal Nehru Centre for Advanced Scientific Research, Jakkur, Bangalore 560 064, India; rahul@iisc.ac.in
}

(KPZ) equation [33-36], a stochastic PDE (SPDE), in which the height field $h(\mathbf{x}, t)$ is kinetically roughened. The elucidation of the statistics of $h(\mathbf{x}, t)$ in the KPZ SPDE has played a central role in nonequilibrium statistical mechanics, in general, and interface-growth phenomena, in particular. Early KPZ studies $[33,34]$ have concentrated on height-field correlations, the width $w(L, t)$ of the fluctuating KPZ interface, and their power-law dependences on the linear system size $L$ and time $t$, for large $L$ and $t$ (see below); for the 1D case, several results can be obtained analytically. The universality of the power-law exponents has been demonstrated by explicit numerical calculations, e.g., in the polynuclear growth (PNG) model, directed polymers in random media (DPRM), and the asymmetric simple exclusion process (ASEP), and by experiments in turbulent liquid crystals [37-39], all of which lie (in suitable parameter regimes) in the KPZ universality class. The seminal work of Prähofer and Spohn work (recently referred to as "the second KPZ Revolution" [35]) on the PNG model [40] and the mathematical studies of Johansson [41] and Baik, Deift, and Johansson [42] has led to a new set of studies of the 1D KPZ universality class [36,43-48], which have led to the remarkable result that, at a point $x$ and at large times $t$,

$h(x, t)-h(x, 0) \approx v_{\infty} t+(\Gamma t)^{\beta_{\mathrm{KPZ}}} \chi_{\beta}+o\left(t^{\beta_{\mathrm{KPZ}}}\right)$, for $t \rightarrow \infty$,

where $v_{\infty}$ and $\Gamma$ are model-dependent constants (see the Supplemental Material [49]), the exponent $\beta_{\mathrm{KPZ}}=1 / 3$, and $\chi_{\beta}$ is a random variable distributed according to the TracyWidom (TW) distribution for the Gaussian orthogonal ensemble $(\mathrm{GOE})(\beta=1)$ and for the Gaussian unitary ensemble (GUE) $(\beta=2)$, familiar from the theory of random matrices [50-52], or the Baik-Rains (BR $\left.F_{0}\right)$ distribution [53] $(\beta=0)$; the value of $\beta$ depends on the initial condition. We show, by extensive direct numerical simulations (DNSs), that the 
result (1) holds for the NESS of the 1D KS PDE. Thus, the correspondence between the statistical properties of these states, in the 1D KS (PDE) and their counterparts in the 1D KPZ (SPDE), does not stop at the simple correlation functions, investigated so far [27-30]; we demonstrate that this correspondence includes the universal limit distributions obtained in "the second KPZ Revolution" [35].

The KS PDE, which predates the KPZ SPDE, is

$$
\partial_{t} h(\mathbf{x}, t)+\Delta h(\mathbf{x}, t)+\Delta^{2} h(\mathbf{x}, t)+\frac{1}{2}[\nabla h(\mathbf{x}, t)]^{2}=0,
$$

where $\nabla \equiv \partial / \partial \mathbf{x}, \partial_{t} \equiv \partial / \partial t, \Delta \equiv \nabla^{2}$, and $h, \mathbf{x}$, and $t$ have been scaled such that the linear system size $L$ is the only control parameter. The dynamical and long-wavelength properties of the 1D KS PDE have been explored via DNSs in Refs. [27-29,55,56]; several mathematical results have been obtained in Refs. [57-59].

The 1D KPZ SPDE is

$$
\begin{aligned}
\partial_{t} h(x, t) & =v \Delta h(x, t)+\frac{\lambda}{2}[\nabla h(x, t)]^{2}+\eta, \\
\left\langle\eta(x, t) \eta\left(x^{\prime}, t^{\prime}\right)\right\rangle & =D \delta\left(x-x^{\prime}\right) \delta\left(t-t^{\prime}\right),
\end{aligned}
$$

where $v$, the diffusivity, and $\lambda$, the strength of the nonlinearity, are real parameters, and $\eta$ is a zero-mean Gaussian white noise, with variance $D$.

We solve the 1D KS PDE (2), with periodic boundary conditions on a domain of size $L$, by using the pseudospectral method [60-65] and the $2 / 3$ dealiasing rule. For time marching we use the fourth-order exponential time-differencing Runge-Kutta scheme ETDRK4 [66,67]. For reliable statistics, it is important to carry out long simulations with large values of $L$; we report results with $L=2^{20}$, by far the highest spatial resolution that has been used for a DNS of the 1D KS PDE (2); for this we have developed a CUDA C code that runs very efficiently on a GPU cluster with NVIDIA Tesla K80 accelerators.

From our DNSs we compute $h(x, t)$ for six different kinds of initial conditions, IC1-IC6, which we depict by plots of $h(x, 0)$ vs $x$ in Figs. 1(a), 1(e) 1(i), 1(m), 1(q), and 1(u); we show the short-time spatiotemporal evolution of $h(x, t)$, in the interval $x \in[-100,100]$, in Figs. 1(b), 1(f) 1(j), 1(n), $1(\mathrm{r})$, and 1(v) (see the videos V1-V6 in the Supplemental Material [49]). We choose these ICs to mimic the effect of wedge, flat, stationary, wedge-to-stationary, wedge-to-flat, and flat-to-stationary geometries in the ASEP model, which are listed in Refs. $[46,68,69]$ as initial conditions for six different subclasses of the 1D KPZ universality class. Previous numerical studies $[28,29]$ of the $1 \mathrm{D}$ KS PDE have shown that two-point, equal-time height-field correlations show the scaling behaviors of their 1D KPZ SPDE counterparts for times greater than a crossover time $t_{c} \simeq 18700$ and lengths larger than the crossover size $L_{c} \simeq 3600$. Therefore, we use a very large system size $L=2^{20}$ and very long simulation times $t_{\max } \geqslant 2 \times 10^{5}$ (see the Supplemental Material [49]).

Our results for two-point height correlation functions are consistent with those of earlier investigations $[28,29]$ of the statistical properties of the spatiotemporally chaotic state of the 1D KS PDE: We show, e.g., the equal-time compensated spectrum $k^{2} E(k)=\left\langle L \tilde{h}(k, t) \tilde{h}^{*}(k, t)\right\rangle_{t}$, where $\langle\cdot\rangle_{t}$ is the time average, $\tilde{h}(k, t)$ is the spatial Fourier transform of $h(x, t)$, and $k$ is the wave number, in Fig. S1 of the Supplemental Material [49]. In addition, we calculate the time-dependent, two-point correlation function $S(k, \delta t)=\left\langle k^{2} \tilde{h}\left(k, t_{0}\right) \tilde{h}^{*}\left(k, t_{0}+\delta t\right)\right\rangle_{t_{0}}$ in Fig. 2, for the IC3 initial condition. We find that the imaginary part of $S(k, \delta t)$ fluctuates around zero and its magnitude is much smaller than that of its real part, which we plot in Fig. 2. Our data are consistent with the scaling form of $S(k, \delta t)$ (orange curve in Fig. 2), which has been obtained analytically by Prähofer and Spohn [54] for the 1D KPZ SPDE.

The scaling properties of the interface width $w(L, t)$ distinguish different universality classes in interface-growth models,

$$
w(l, t)=\left(\left\langle\left[\Delta_{l} h(x, t)\right]^{2}\right\rangle_{x, l}\right)^{1 / 2},
$$

with $\Delta_{l} h(x, t)=h(x, t)-h(x, 0)-\langle h(x, t)-h(x, 0)\rangle_{x, l}$ and $\langle\cdot\rangle_{x, l}$ the spatial average over a region of spatial extent $l$. For $t \gg 1$ in the $1 \mathrm{D} \mathrm{KPZ}$ equation, $w(L, t) \sim t^{\beta}$. Before crossover occurs in systems with $L>L_{c}$, the exponent $\beta$ assumes the value $\beta_{\mathrm{EW}}=1 / 4$, which is the Edwards-Wilkinson (EW) result $[34,70]$ for the linear SPDE with $\lambda=0$ in Eq. (3); finally, $\beta$ assumes the KPZ value $\beta_{\mathrm{KPZ}}=1 / 3$ in the NESS (for $t>t_{c}$ ). Moreover, the growing KPZ surface involves the length scale $\mathcal{L}(t) \sim t^{1 / z}$, where the dynamic exponent $z=$ $3 / 2$, and the width $w(l, t) \sim l^{\alpha}$, for $l \ll \mathcal{L}(t)$, with $\alpha=1 / 2$ [37]. We find from our DNSs of the 1D KS equation that these Family-Vicsek scaling [71] forms are indeed satisfied as we show in Figs. 3(a), 3(c) and 3(e) for IC1-IC3 (see the Supplemental Material [49] for IC4-IC6).

We define

$$
\mu_{n}=\left\langle\left[\Delta_{L} h(x, t)\right]^{n}\right\rangle /\left\langle\left[\Delta_{L} h(x, t)\right]^{2}\right\rangle^{n / 2}-3 \delta_{n, 4} ;
$$

for $n=3(n=4), \mu_{n}$ is the skewness (kurtosis); we plot $\mu_{3}$ and $\mu_{4}$ versus time $t$ in the right panel of Fig. 3; for each initial condition, IC1-IC6, we average these quantities for 100 surfaces, over a time interval of $10^{4}$, and five independent DNS runs, i.e., our overall sample size is $\simeq 5 \times 10^{8}$ data points. [For our 1D KS, $\mu_{3}<0$ because of the sign of the nonlinear term in Eq. (2); we ignore the sign of $\mu_{3}$ for it can be reversed by the transformation $h(x, t) \rightarrow-h(x, t)$.] In addition, we calculate the probability distribution function (PDF) $\mathrm{P}(\chi)$ of the shifted and rescaled fluctuations, namely, $\chi=\left[h(x, t)-v_{\infty} t\right] /(\Gamma t)^{1 / 3}$, when both $\mu_{3}$ and $\mu_{4}$ are close to their standard values for the relevant TW or BR $F_{0}$ PDFs; for IC2, e.g., we compute $\mathrm{P}(\chi)$ when we have $\mu_{3} \simeq 0.27$ and $\mu_{4} \simeq 0.19$, which are close to the standard values $\mu_{3, \mathrm{GOE}} \simeq$ 0.29 and $\mu_{4, \mathrm{GOE}} \simeq 0.16$, respectively.

For IC1, IC2, IC3, and IC4 we compare, on semilog plots, the PDFs with TW-GUE, TW-GOE, BR $F_{0}$, and $\left(F_{\mathrm{GOE}}\right)^{2}$ [46] in Figs. 1(d), 1(h) 1(l), and 1(p), respectively. For ease of comparison, we show in Fig. 4 that the PDFs we obtain from our DNSs of the 1D KS Eq. (2) are very close to the TW-GUE, TW-GOE, and BR $F_{0}$ PDFs over at least three orders of magnitude. Strictly speaking, we must collect data only from those two points $(x=L / 4,3 L / 4)$ at which the two different types of height profiles meet in cases IC4, IC5, and IC6. However, this leads to inadequate statistics. Therefore, the PDFs of $\chi$ for IC4-IC6, which we show in Figs. 1(p), $1(\mathrm{t})$ and $1(\mathrm{x})$, have been computed by using data from the regions $[7 L / 32,9 L / 32]$ and $[23 L / 32,25 L / 32]$; we see that this averaging procedure already leads to PDFs [Figs. 1(p), 

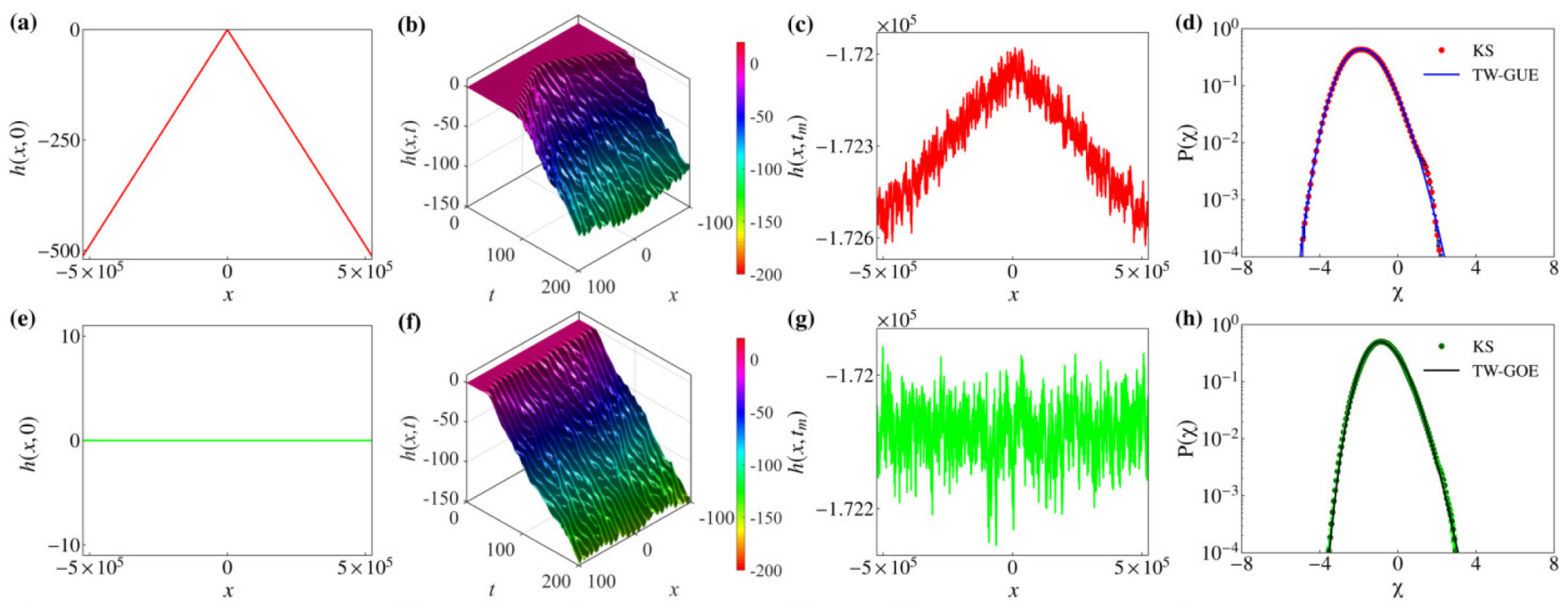
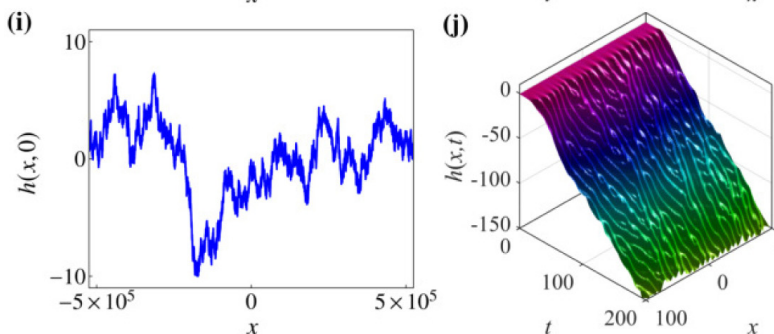

(k)
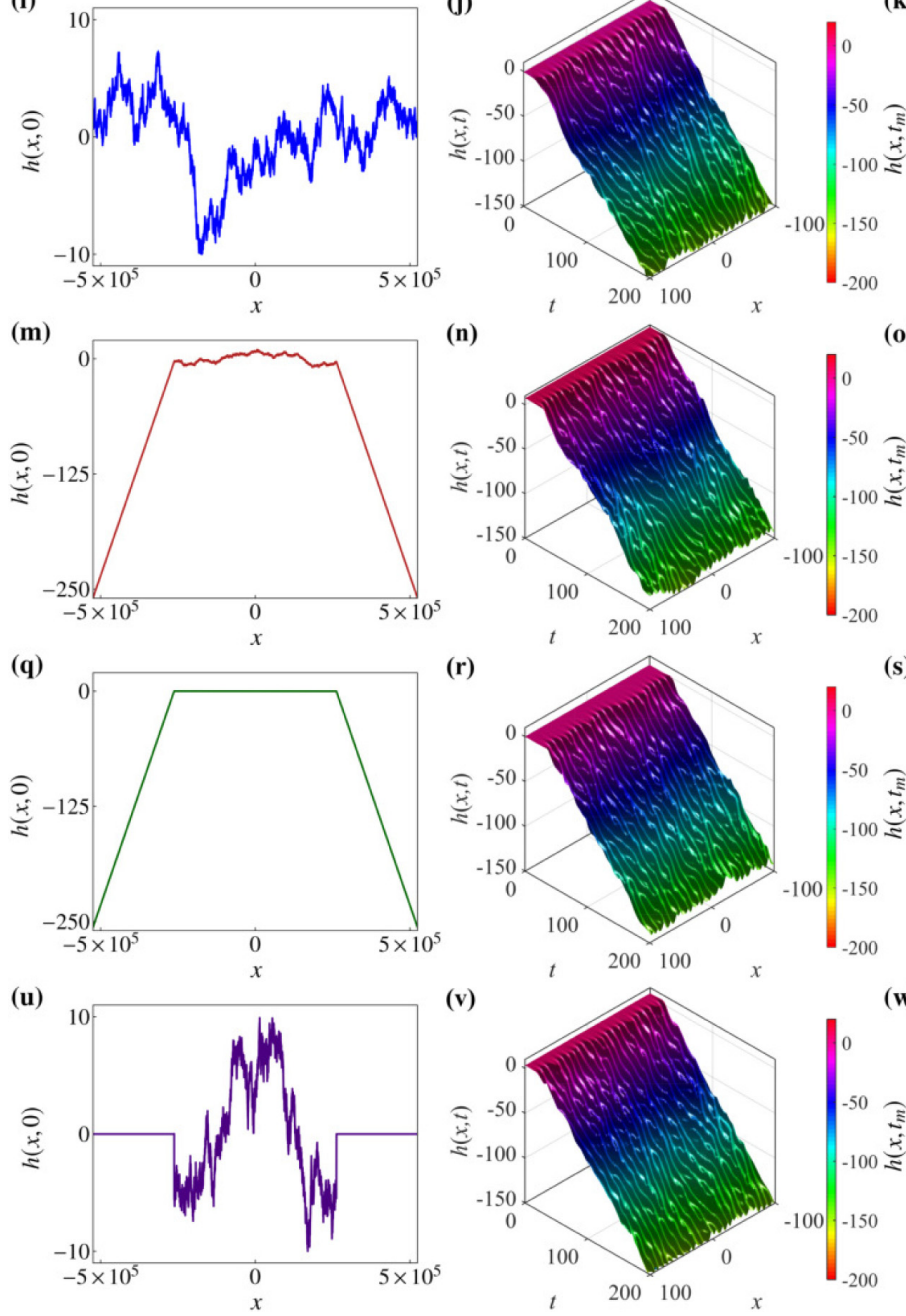
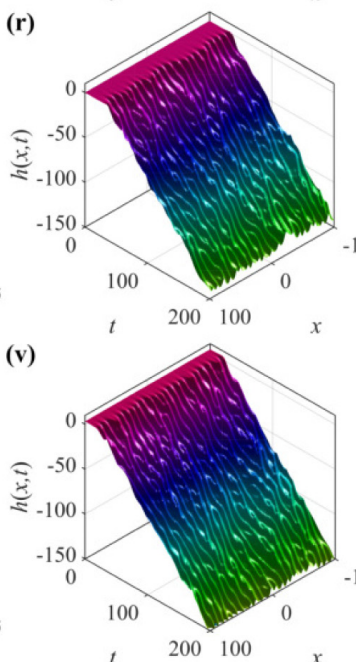

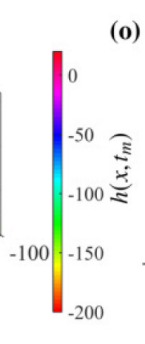

(o)

(w)

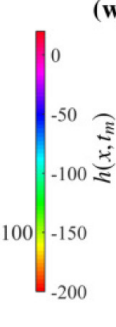

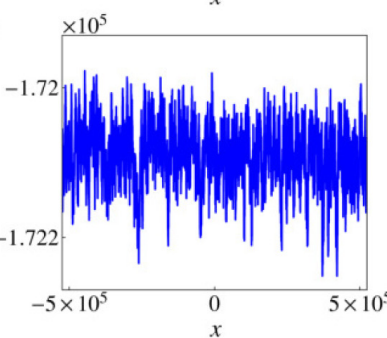
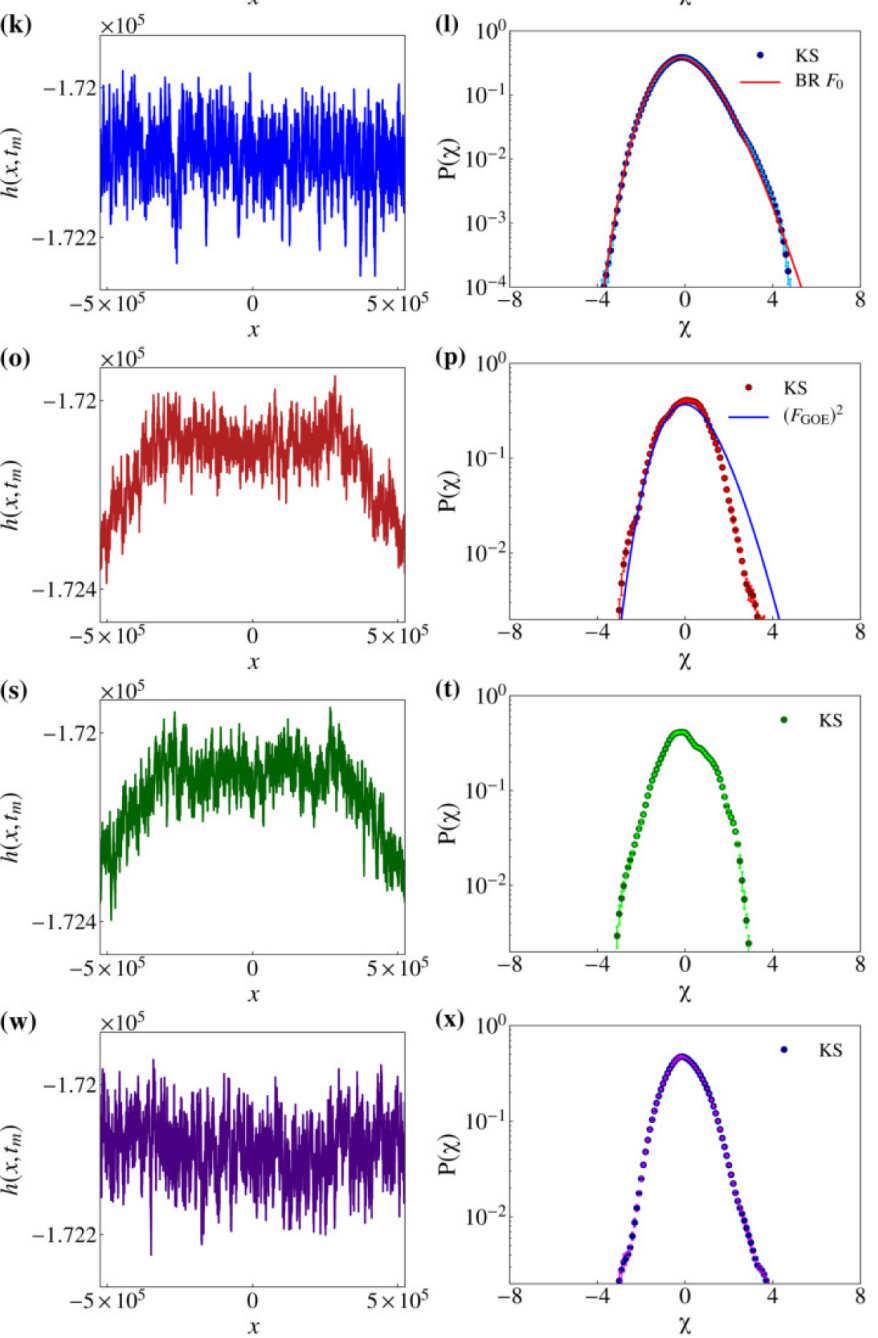

FIG. 1. Plots of $h(x, 0)$ vs $x \in[-L / 2, L / 2]$, with $L=2^{20}$, for the six different initial conditions, IC1-IC6 in (a), (e), (i), (m), (q), and (u), respectively. The short-time spatiotemporal evolution of $h(x, t)$ is shown, in the interval $[-100,100]$, for each one of IC1-IC6 in (b), (f), (j), (n), (r), and (v) (see the videos V1-V6 in the Supplemental Material [49]). The height profiles at time $t_{m}=2 \times 10^{5}$ are plotted in (c), (g), (k), $(\mathrm{o}),(\mathrm{s})$, and $(\mathrm{w})$ for IC1-IC6, respectively; the plots $(\mathrm{d}),(\mathrm{h}),(\mathrm{l}),(\mathrm{p}),(\mathrm{t})$, and (x) display corresponding limit distributions for $\chi(\mathrm{see}$ text) in the NESSs; and in (d), (h), (l), and (x) we plot TW-GUE, TW-GOE, BR $F_{0}$, and $\left(F_{\mathrm{GOE}}\right)^{2}$ distributions to compare them with data from our DNSs. The error bars on $\mathrm{P}(\chi)$ are smaller than the sizes of our symbols. 


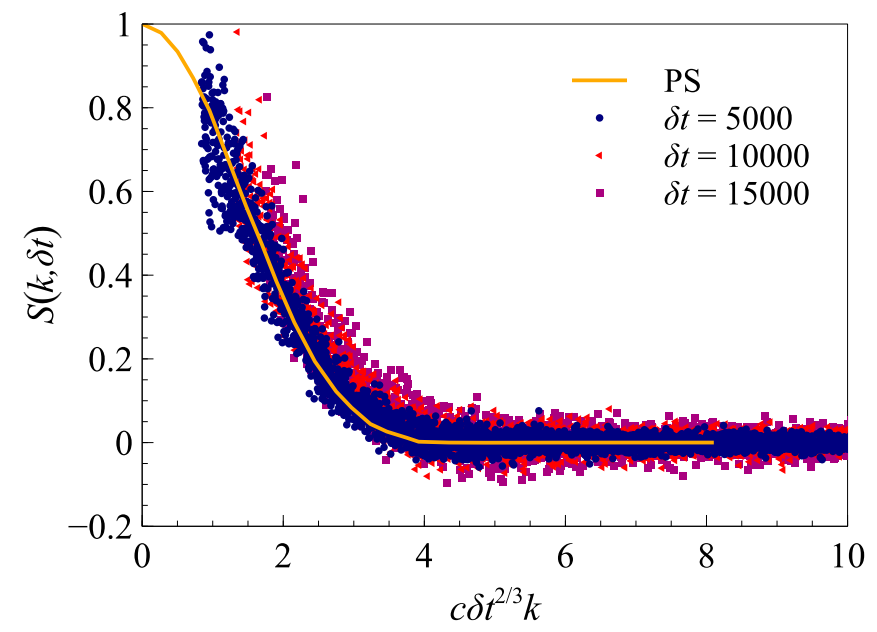

FIG. 2. Plot of the scaling form of the Fourier transform of the two-point time-dependent correlation function $S(k, \delta t) \mathrm{vs} c \delta t^{2 / 3} k$, with the nonuniversal $c=1.6$, computed for IC3 [see Fig. 1(i)]. We plot $S(k, \delta t)$ for three different values of $\delta t$; we also show, for comparison, the theoretical result (orange curve PS) obtained by Prähofer and Spohn [54] for the 1D KPZ equation.
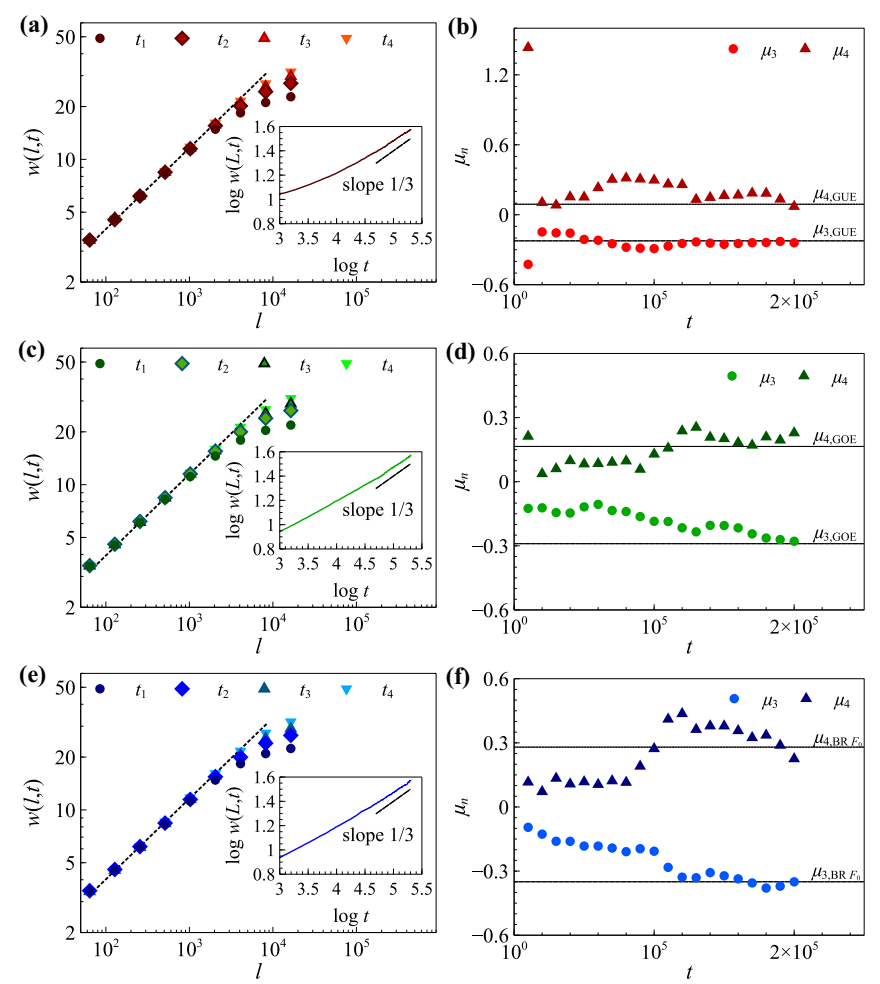

FIG. 3. Family-Vicsek scaling [71]: (a), (c), and (e) show, for IC1-IC3, respectively, plots of $w(l, t)$ vs $l$, for $l \ll L$, and $w(L, t)$ vs $t$ (in the insets); $t_{1}=5 \times 10^{4}, t_{2}=10^{5}, t_{3}=1.5 \times 10^{5}$, and $t_{4}=$ $2 \times 10^{5}$. The dotted lines are $\log$-log fits for $w(l, t)=A l^{\alpha}$, with $\alpha=0.46 \pm 0.07$ for IC1-IC3. In (b), (d), and (f), we plot, for IC1IC3, respectively, the skewness $\mu_{3}$ and the kurtosis $\mu_{4}$ (see text) vs the time $t$; black lines indicate their large- $t$ values for TW-GUE, TW-GOE, and BR $F_{0}$ PDFs in (b), (d), and (f). (See the Supplemental Material [49] for similar plots for IC4-IC6.)

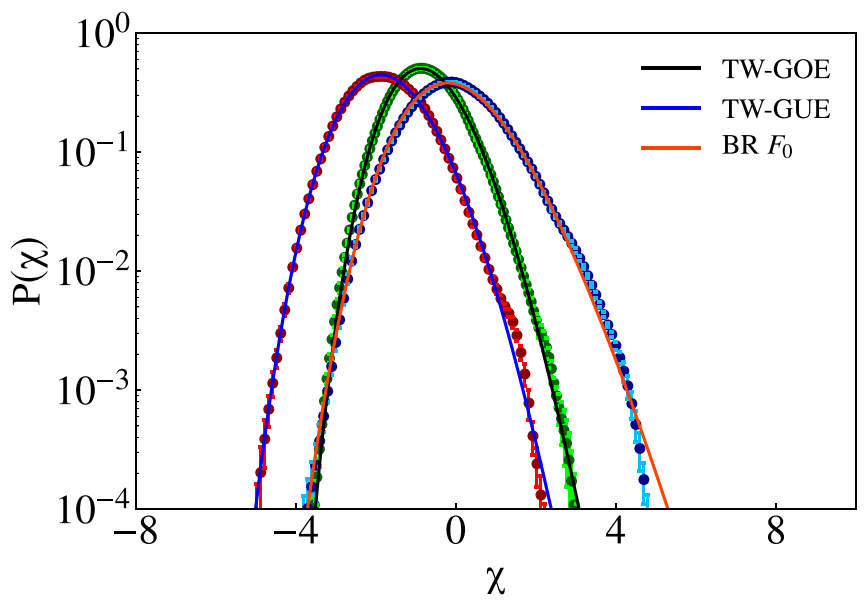

FIG. 4. Semilog plots of the PDFs $\mathrm{P}(\chi)$ from our DNSs for IC1, IC2, and IC3; we compare these with the Tracy-Widom distributions, TW-GUE and TW-GOE, and the Baik-Rains distributions (BR $F_{0}$ ).

1(t) and $1(\mathrm{x})$ ] that are distinctly different from TW-GUE, TW-GOE, and BR $F_{0}$ distributions.

The TW distributions, for IC1 and IC2 initial conditions in the 1D KPZ equation, have been studied in the context of $N \times N$ GOE $(\beta=1)$ and GUE $(\beta=2)$ random matrices. The largest eigenvalue (after scaling with $N$ ) $\Lambda$ of such random matrices is

$$
\Lambda=\sqrt{2}+\frac{1}{\sqrt{2}} N^{-2 / 3} \chi_{\beta}
$$

where $\chi_{\beta}$ has the PDF [72]

$$
\begin{aligned}
& \mathrm{P}(\Lambda, N) \\
& \quad \approx \begin{cases}\exp \left[-\beta N^{2} \phi_{-}(\Lambda)\right], & \Lambda<\sqrt{2},|\Lambda-\sqrt{2}| \sim O(1), \\
\sqrt{2} N^{2 / 3} \mathrm{P}_{\mathrm{TW}, \beta}\left(\chi_{\beta}\right), & |\Lambda-\sqrt{2}| \sim O\left(N^{-2 / 3}\right), \\
\exp \left[-\beta N \phi_{+}(\Lambda)\right], & \Lambda>\sqrt{2},|\Lambda-\sqrt{2}| \sim O(1),\end{cases}
\end{aligned}
$$

where $\mathrm{P}_{\mathrm{TW}, \beta}\left(\chi_{\beta}\right)$ denotes TW distributions, and the right and left large-deviation functions (LDFs) $\phi_{+}(\Lambda)$ and $\phi_{-}(\Lambda)$, respectively, display the following asymptotic behaviors,

$$
\begin{aligned}
& \phi_{-}(\Lambda) \approx \frac{1}{6 \sqrt{2}}(\sqrt{2}-\Lambda)^{3}, \quad \Lambda \rightarrow-\infty, \\
& \phi_{+}(\Lambda) \approx \frac{2^{7 / 4}}{3}(\Lambda-\sqrt{2})^{3 / 2}, \quad \Lambda \rightarrow+\infty .
\end{aligned}
$$

The LDFs, which yield the probabilities of atypically large fluctuations, match smoothly with the tails of $\mathrm{P}_{\mathrm{TW}, \beta}\left(\chi_{\beta}\right)$. Because of different behaviors of the tails of $\mathrm{P}(\Lambda, N)$, a thirdorder transition [72] can be associated with $\Lambda$ at $\Lambda_{c}=\sqrt{2}$ by defining the free energy $\propto \ln F_{\beta}(\Lambda, N), F_{\beta}(\Lambda, N)$ being the cumulative density function (CDF) for $\Lambda$, for which we have [72]

$$
\lim _{N \rightarrow \infty}-\frac{1}{N^{2}} \ln F_{\beta}(\Lambda, N)= \begin{cases}\phi_{-}(\Lambda), & \Lambda<\sqrt{2} \\ 0, & \Lambda>\sqrt{2}\end{cases}
$$

Similarly, we define, for the KS initial conditions IC1 and IC2, the free-energy function $\mathcal{F}(\bar{h})$ for $t, L \rightarrow \infty$ as 
follows,

$$
\mathcal{F}(\bar{h})=\lim _{t, L \rightarrow \infty}-\frac{1}{t^{2}} \ln F(\chi, t),
$$

where $\bar{h}=h(x, t) / t$ and $F(\chi, t)$ is the CDF for $\chi$ at time $t$. Therefore, for IC1 and IC2, we should obtain a third-order phase transition for $\bar{h}$ at the critical value $\bar{h}_{c}=v_{\infty}$; an explicit demonstration requires much better statistics for $\mathrm{P}(\chi)$ than is possible with our DNS.

We have shown, by extensive pseudospectral DNSs of the 1D KS deterministic PDE, that the statistical properties of its spatiotemporally chaotic NESS are in the 1D KPZ universality class. This is not limited, merely, to the power-law forms of simple correlation functions and the width of the interface. It includes, in addition, (a) the complete scaling form for the two-point time-dependent correlation function $S(k, \delta t)$ (Fig. 2), (b) the skewness and kurtosis shown in Fig. 2, and (c) most important of all, the universal limit distributions in Fig. 1, obtained in "the second KPZ revolution" [35]. We obtain such results for a spatiotemporally chaotic NESS of a deterministic PDE. We conjecture that similar conclusions should ensue for the phase-chaos regime of the 1D complexGinzburg-Landau equation [25]. Such studies are also being pursued for the 1D Calogero-Moser model [73].

We thank Jaya Kumar Alageshan, R. Basu, M. Brachet, P. Ferrari, K. Khanin, and K. A. Takeuchi for discussions, T. Imamura for discussions and for sending us the Baik-Rains $\mathrm{CDF}$, and the National Mathematics Initiative (NMI), DST, UGC, and CSIR (India) for support.
[1] S. F. Edwards, J. Fluid Mech. 18, 239 (1964).

[2] D. Forster, D. R. Nelson, and M. J. Stephen, Phys. Rev. A 16, 732 (1977).

[3] C. DeDominicis and P. C. Martin, Phys. Rev. A 19, 419 (1979).

[4] J. D. Fournier and U. Frisch, Phys. Rev. A 28, 1000 (1983).

[5] V. Yakhot and S. A. Orszag, J. Sci. Comput. 1, 3 (1986).

[6] C.-Y. Mou and P. B. Weichman, Phys. Rev. E 52, 3738 (1995).

[7] J. K. Bhattacharjee, J. Phys. A: Math. Gen. 21, L551 (1988).

[8] L. T. Adzhemyan, N. V. Antonov, and A. N. Vasiliev, Phys. Usp. 39, 1193 (1996).

[9] L. T. Adzhemyan, N. V. Antonov, and A. N. Vasiliev, Field Theoretic Renormalization Group in Fully Developed Turbulence (Gordon and Breach, London, 1999).

[10] A. Sain, Manu, and R. Pandit, Phys. Rev. Lett. 81, 4377 (1998).

[11] L. Biferale, M. Cencini, A. S. Lanotte, M. Sbragaglia, and F. Toschi, New J. Phys. 6, 37 (2004).

[12] A. N. Kolmogorov, Dokl. Akad. Nauk SSSR 30, 301 (1941).

[13] A. N. Kolmogorov, Dokl. Akad. Nauk SSSR 31, 538 (1941).

[14] U. Frisch, Turbulence: The Legacy of A. N. Kolmogorov (Cambridge University Press, Cambridge, U.K., 1995).

[15] Turbulence and Predictability in Geophysical Fluid Dynamics and Climate Dynamics, Proceedings of the International School of Physics "Enrico Fermi", Course 88, Varenna, 1983, edited by M. Ghil, R. Benzi, and G. Parisi (North-Holland, Amsterdam, 1985).

[16] R. Benzi, G. Paladin, G. Parisi, and A. Vulpiani, J. Phys. A: Math. Gen. 17, 3521 (1984).

[17] R. Benzi and U. Frisch, Scholarpedia 5, 3439 (2010).

[18] C. Meneveau and K. R. Sreenivasan, J. Fluid Mech. 224, 429 (1991).

[19] Y. Kuramoto and T. Tsuzuki, Prog. Theor. Phys. 55, 356 (1976).

[20] G. Sivashinsky, Acta Astronaut. 4, 1177 (1977).

[21] G. I. Sivashinsky and D. M. Michelson, Prog. Theor. Phys. 63, 2112 (1980)

[22] C. Ruyer-Quil and P. Manneville, Eur. Phys. J. B 6, 277 (1998).

[23] Macroscopic Modelling of Turbulent Flows, Lecture Notes in Physics Vol. 230 (Springer, Berlin, 1985).

[24] L.-H. Chen and H.-C. Chang, Chem. Eng. Sci. 41, 2477 (1986).

[25] G. Grinstein, C. Jayaprakash, and R. Pandit, Physica D 90, 96 (1996).
[26] V. Yakhot, Phys. Rev. A 24, 642 (1981).

[27] J. M. Hyman, B. Nicolaenko, and S. Zaleski, Physica D 23, 265 (1986).

[28] K. Sneppen, J. Krug, M. H. Jensen, C. Jayaprakash, and T. Bohr, Phys. Rev. A 46, R7351 (1992).

[29] F. Hayot, C. Jayaprakash, and C. Josserand, Phys. Rev. E 47, 911 (1993).

[30] C. Jayaprakash, F. Hayot, and R. Pandit, Phys. Rev. Lett. 71, 12 (1993).

[31] B. M. Boghosian, C. C. Chow, and T. Hwa, Phys. Rev. Lett. 83, 5262 (1999).

[32] A. Kalogirou, E. E. Keaveny, and D. T. Papageorgiou, Proc. R. Soc. London, Ser. A 471, 20140932 (2015).

[33] M. Kardar, G. Parisi, and Y.-C. Zhang, Phys. Rev. Lett. 56, 889 (1986).

[34] T. Halpin-Healy and Y.-C. Zhang, Phys. Rep. 254, 215 (1995).

[35] T. Halpin-Healy and K. A. Takeuchi, J. Stat. Phys. 160, 794 (2015).

[36] J. Quastel and H. Spohn, J. Stat. Phys. 160, 965 (2015).

[37] K. A. Takeuchi, M. Sano, T. Sasamoto, and H. Spohn, Sci. Rep. 1, 34 (2011).

[38] K. A. Takeuchi and M. Sano, J. Stat. Phys. 147, 853 (2012).

[39] K. A. Takeuchi, Phys. Rev. Lett. 110, 210604 (2013).

[40] M. Prähofer and H. Spohn, Phys. Rev. Lett. 84, 4882 (2000).

[41] K. Johansson, Commun. Math. Phys. 209, 437 (2000).

[42] J. Baik, P. Deift, and K. Johansson, J. Am. Math. Soc. 12, 1119 (1999).

[43] T. Sasamoto and H. Spohn, Phys. Rev. Lett. 104, 230602 (2010).

[44] P. Calabrese and P. Le Doussal, Phys. Rev. Lett. 106, 250603 (2011).

[45] T. Imamura and T. Sasamoto, Phys. Rev. Lett. 108, 190603 (2012).

[46] I. Corwin, Random Matrices: Theory Appl. 01, 1130001 (2012)

[47] T. Halpin-Healy and Y. Lin, Phys. Rev. E 89, 010103(R) (2014).

[48] A. A. Saberi, H. Dashti-Naserabadi, and J. Krug, Phys. Rev. Lett. 122, 040605 (2019).

[49] See Supplemental Material at http://link.aps.org/supplemental/ 10.1103/PhysRevE.101.030103 for details about KPZ scaling 
parameters, the energy spectrum, Family-Vicsek scaling for IC4-6, direct numerical simulations, and URLs of our videos of the spatiotemporal evolution of the height field for IC1-6.

[50] C. A. Tracy and H. Widom, Commun. Math. Phys. 159, 151 (1994).

[51] F. Bornemann, Math. Comput. 79, 871 (2010).

[52] Marco (2020), Approximation for the Tracy-Widom laws, MATLAB Central File Exchange, https://www.mathworks. com/matlabcentral/fileexchange/44711-approximation-forthe-tracy-widom-laws.

[53] J. Baik and E. M. Rains, J. Stat. Phys. 100, 523 (2000).

[54] M. Prähofer and H. Spohn, J. Stat. Phys. 115, 255 (2004).

[55] J. M. Hyman and B. Nicolaenko, Physica D 18, 113 (1986).

[56] I. G. Kevrekidis, B. Nicolaenko, and J. C. Scovel, SIAM J. Appl. Math. 50, 760 (1990).

[57] P. Collet, J.-P. Eckmann, H. Epstein, and J. Stubbe, Commun. Math. Phys. 152, 203 (1993).

[58] M. Jolly, I. Kevrekidis, and E. Titi, Physica D 44, 38 (1990).

[59] R. Conte and M. Musette, J. Phys. A: Math. Gen. 22, 169 (1989).

[60] C. Canuto and A. Quarteroni, CALCOLO 18, 197 (1981).
[61] C. Canuto, M. Y. Hussaini, A. Quarteroni, and T. A. Zang, Spectral Methods (Springer, Berlin, 2006).

[62] L. N. Trefethen, Spectral Methods in MATLAB (SIAM, Philadelphia, 2000).

[63] M. K. Verma, Physica A 277, 359 (2000).

[64] A. Giacometti and M. Rossi, Phys. Rev. E 63, 046102 (2001).

[65] L. Giada, A. Giacometti, and M. Rossi, Phys. Rev. E 65, 036134 (2002).

[66] A.-K. Kassam and L. N. Trefethen, SIAM J. Sci. Comput. 26, 1214 (2005).

[67] S. Cox and P. Matthews, J. Comput. Phys. 176, 430 (2002).

[68] A. Borodin, P. L. Ferrari, and T. Sasamoto, Commun. Pure Appl. Math. 61, 1603 (2008).

[69] I. Corwin, P. L. Ferrari, and S. Péché, J. Stat. Phys. 140, 232 (2010).

[70] S. F. Edwards and D. Wilkinson, Proc. R. Soc. London, Ser. A 381, 17 (1982).

[71] F. Family and T. Vicsek, J. Phys. A: Math. Gen. 18, L75 (1985).

[72] S. N. Majumdar and G. Schehr, J. Stat. Mech.: Theory Exp. (2014) P01012.

[73] S. Agarwal, M. Kulkarni, and A. Dhar, J. Stat. Phys. 176, 1463 (2019). 